\title{
Quasi-geostrophic equations, nonlinear Bernstein inequalities and $\alpha$-stable processes
}

\author{
Diego Chamorro and Pierre Gilles Lemarié-Rieusset
}

\begin{abstract}
We prove some functional inequalities for the fractional differentiation operator $(-\Delta)^{\alpha}$ through the formalism of semi-groups. This gives us an estimate of the regularity of Marchand's weak solutions for the dissipative quasi-geostrophic equation.
\end{abstract}

\section{Introduction}

In this paper, we are interested in the regularity of weak solutions of the dissipative quasi-geostrophic equation $\left(Q G_{\alpha}\right)$, a generalization of the quasi-geostrophic equation $(Q G)$ which is related to fluid mechanics, [13], and whose mathematical study was initiated by Constantin, Majda and Tabak in 1994 ([5]). The quasi-geostrophic equation $(Q G)$ describes the evolution of a function $\theta(t, x), t>0, x \in \mathbb{R}^{2}$, as

$$
\left\{\begin{aligned}
\partial_{t} \theta+\vec{u} \cdot \vec{\nabla} \theta & =0, \\
\vec{u} & =\left(-R_{2} \theta, R_{1} \theta\right), \\
\theta(0, .) & =\theta_{0},
\end{aligned}\right.
$$

where $R_{i}$ is the Riesz transform, $R_{i}=\frac{\partial_{i}}{\sqrt{-\Delta}}$ (so that the vector field $\vec{u}$ is divergencefree: $\operatorname{div} \vec{u}=0$ ).

Throughout the paper, we will denote $\sqrt{-\Delta}$ by $\Lambda$ (this is Calderón's operator). For $0<\alpha \leq 1$, the dissipative quasi-geostrophic equation $\left(Q G_{\alpha}\right)$ is the equation $(Q G)$ penalized by a dissipative term $-\Lambda^{2 \alpha} \theta$ :

$$
\left\{\begin{aligned}
\partial_{t} \theta+\vec{u} \cdot \vec{\nabla} \theta & =-\Lambda^{2 \alpha} \theta, \\
\vec{u} & =\left(-R_{2} \theta, R_{1} \theta\right), \\
\theta(0, .) & =\theta_{0} .
\end{aligned}\right.
$$

Mathematics Subject Classification (2010): Primary 35Q35; Secondary 58J35.

Keywords: Besov spaces, semi-groups, Bernstein inequalities, quasi-geostrophic equation, stratified Lie groups, $\alpha$-stable processes. 
In order to deal with irregular solutions, we rewrite the advection term $\vec{u} . \vec{\nabla} \theta$ as $\operatorname{div}(\theta \vec{u})$ :

$$
\left\{\begin{aligned}
\partial_{t} \theta+\operatorname{div}(\theta \vec{u}) & =-\Lambda^{2 \alpha} \theta, \\
\vec{u} & =\left(-R_{2} \theta, R_{1} \theta\right), \\
\theta(0, .) & =\theta_{0} .
\end{aligned}\right.
$$

In 1995, Resnick [15] proved the existence of weak solutions of the equation (1.3) for $\theta_{0} \in L^{2}\left(\mathbb{R}^{2}\right)$; these solutions satisfy the inequality

$$
\text { for } t>0, \quad\|\theta(t, .)\|_{2}^{2}+2 \int_{0}^{t} \int\left|\Lambda^{\alpha} \theta\right|^{2} d x d s \leq\left\|\theta_{0}\right\|_{2}^{2},
$$

so that $\theta \in L_{t}^{\infty} L^{2} \cap L_{t}^{2} \dot{H}^{\alpha}$, where $\dot{H}^{\alpha}$ is a homogeneous Sobolev space.

In 2008, Marchand [12] studied the case of an initial value $\theta_{0} \in L^{p}$; he proved the existence of weak solutions to equation (1.3) when $p \geq 4 / 3$. Moreover, when $p \geq 2$, Marchand's solutions satisfy the inequality

$$
\text { for } t>0, \quad\|\theta(t, .)\|_{p}^{p}+p \int_{0}^{t} \int \theta|\theta|^{p-2} \Lambda^{2 \alpha} \theta d x d s \leq\left\|\theta_{0}\right\|_{p}^{p},
$$

where the double integral gives a nonnegative contribution, as shown by Córdoba's inequality [6], [10]:

$$
2 \int\left|\Lambda^{\alpha}\left(|\theta|^{p / 2}\right)\right|^{2} d x \leq p \int \theta|\theta|^{p-2} \Lambda^{2 \alpha} \theta d x
$$

However, the regularity of Marchand's solutions remained unclear.

In this paper, we will establish the regularity of Marchand's solutions in terms of a norm in a Besov space. More precisely, we shall establish a variant of Córdoba's inequality and get that, for $2 \leq p<\infty$ and $0<\alpha<1$,

$$
\|\theta\|_{\dot{B}_{p}^{2 \alpha / p, p}}^{p} \leq C_{p} \int \theta|\theta|^{p-2} \Lambda^{2 \alpha} \theta d x
$$

and for $2 \leq p<\infty$,

$$
\|\theta\|_{\dot{B}_{p}^{2 / p, \infty}}^{p} \leq C_{p} \int \theta|\theta|^{p-2}(-\Delta) \theta d x
$$

where $\dot{B}_{p}^{2 \alpha / p, p}$ and $\dot{B}_{p}^{2 / p, \infty}$ are homogeneous Besov spaces. Our method gives us a new proof of a nonlinear Bernstein inequality given by Danchin [8]: for $\theta \in L^{p}\left(\mathbb{R}^{n}\right)$ such that its Fourier transform $\hat{\theta}(\xi)$ is supported in the annulus $1 / 2 \leq|\xi| \leq 2$, we have, for $1<p<\infty$,

$$
A\|\theta\|_{p}^{p} \leq\left\|\vec{\nabla}\left(|\theta|^{p / 2}\right)\right\|_{2}^{2} \leq B\|\theta\|_{p}^{p},
$$

where the constants $A$ and $B$ are positive and depend only on $p$ and on the dimension $n$. 
We will mainly apply general results from the theory of semi-groups to the semigroup $e^{-t \Lambda^{2 \alpha}}$. This is a symmetric diffusion semi-group (in the sense of Stein [19]), and we will use a representation of the semi-group as a barycentric mean of heat kernels through a formula derived from the theory of $\alpha$-stable processes [22]. For instance, when $\alpha=1$, we have $e^{-t \Lambda^{2}}=e^{t \Delta}$ (the heat kernel); for $\alpha=1 / 2$, we have $e^{-t \Lambda}=P_{t}$, the Poisson semi-group. In dimension $1, e^{-|\xi|}$ is the Fourier transform of $\frac{1}{\pi} \frac{1}{1+x^{2}}$; we write

$$
\frac{1}{\pi} \frac{1}{1+x^{2}}=\frac{1}{\pi} \int_{0}^{\infty} e^{-\sigma} e^{-\sigma x^{2}} d \sigma=\frac{1}{2 \pi} \int_{0}^{\infty} e^{-\frac{1}{2 \sigma}} e^{-\frac{x^{2}}{2 \sigma}} \frac{d \sigma}{\sigma^{2}}
$$

and we get

$$
e^{-|\xi|}=\frac{1}{\sqrt{2 \pi}} \int_{0}^{\infty} e^{-\frac{1}{2 \sigma}} e^{-\sigma \frac{\xi^{2}}{2}} \frac{d \sigma}{\sigma^{3 / 2}}
$$

and finally

$$
e^{-t \Lambda}=\frac{1}{\sqrt{2 \pi}} \int_{0}^{\infty} e^{-\frac{1}{2 \sigma}} e^{\sigma \frac{t^{2}}{2} \Delta} \frac{d \sigma}{\sigma^{3 / 2}} .
$$

We shall use a generalization of (1.12) to the case of $e^{-t \Lambda^{2 \alpha}}$.

\section{One-dimensional stable distributions}

The aim of this section is to recall the useful following representation:

Proposition 2.1. For $0<\alpha<1$, there exists a probability measure $d \mu_{\alpha}$ concentrated on $[0,+\infty)$ such that for all $x \in \mathbb{R}$ we have

$$
e^{-|x|^{2 \alpha}}=\int_{0}^{+\infty} e^{-\sigma x^{2}} d \mu_{\alpha}(\sigma)
$$

Corollary 2.2. Let $\Lambda=\sqrt{-\Delta}$ be the Calderón operator on $\mathbb{R}^{n}$ and let $e^{t \Delta}$ be the heat kernel on $\mathbb{R}^{n}$. Then the operator $e^{-t \Lambda^{2 \alpha}}(t \geq 0,0<\alpha<1)$ may be represented as

$$
e^{-t \Lambda^{2 \alpha}}=\int_{0}^{+\infty} e^{\sigma t^{1 / \alpha} \Delta} d \mu_{\alpha}(\sigma)
$$

Formula (2.1) is well known. See for instance Proposition 1.2.12 in [18]. Due to a celebrated theorem of Bernstein, [2], it amounts to say that the function $x>0 \mapsto e^{-|x|^{\alpha}}$ is completely monotone, which is easily checked.

Formula (2.1) is linked to the theory of one-dimensional stable processes. The probability density function $d \mu$ of a random variable $X$ is called $\alpha$-stable [22] if its characteristic function $\chi(\xi)=E\left(e^{i X \xi}\right)=\int e^{i x \xi} d \mu(x)$ is of the form

$$
\chi(\xi)=\left\{\begin{array}{cl}
e^{i m \xi-\sigma^{\alpha}|\xi|^{\alpha}-i \beta \sigma^{\alpha} \xi|\xi|^{\alpha-1} \tan (\pi \alpha / 2)} & \text { if } \alpha \neq 1, \\
e^{i m \xi-\sigma|\xi|+i \beta \sigma \xi \ln |\xi|} & \text { if } \alpha=1 .
\end{array}\right.
$$


The admissible values for the parameters are: $0<\alpha \leq 2$ for the stability index $\alpha, m \in \mathbb{R}$ for the position parameter $m, \sigma \geq 0$ for the scale parameter $\sigma$, and $-1 \leq \beta \leq 1$ for the bias parameter $\beta$. We will write $X \sim S_{\alpha}(m, \sigma, \beta)$.

For $X \sim S_{\alpha}(0, \sigma, 1)$ with $0<\alpha<1$, the function $\chi$ is given by

$$
\chi(\xi)=e^{-\sigma^{\alpha}|\xi|^{\alpha}(1+i \operatorname{sgn}(\xi) \tan (\pi \alpha / 2))} .
$$

If $z^{\alpha}$ is the holomorphic function defined on $\mathbb{C} \backslash \mathbb{R}^{-}$as $z^{\alpha}=|z|^{\alpha} e^{i \alpha \operatorname{Arg}(z)}$ where the argument of $z$ is taken in $(-\pi, \pi)$, we have

$$
(i \xi)^{\alpha}=|\xi|^{\alpha} e^{i \alpha \operatorname{sgn}(\xi) \pi / 2}=\cos (\alpha \pi / 2)|\xi|^{\alpha}(1+i \operatorname{sgn}(\xi) \tan (\alpha \pi / 2)) .
$$

Thus, when $X \sim S_{\alpha}\left((\cos (\alpha \pi / 2))^{-1 / \alpha}, 0,1\right)$, we have $\chi(\xi)=e^{-(i \xi)^{\alpha}}$. For $z=\eta+i \xi$ with $\eta \geq 0$, we have $\left|e^{-z^{\alpha}}\right|=e^{-|z|^{\alpha} \cos (\alpha \operatorname{Arg}(z))} \leq 1$. The Paley-Wiener-Schwartz theorem ensures that the probability density function $d \mu_{\alpha}$ of $X$ is supported on $\mathbb{R}^{+}$ and that, for $z=\xi+i \eta$ with $\eta \geq 0$, we have $e^{(i z)^{\alpha}}=\int_{0}^{+\infty} e^{i \sigma z} d \mu_{\alpha}(\sigma)$. When $z=i x^{2}$, we obtain $e^{-|x|^{2 \alpha}}=\int_{0}^{+\infty} e^{-\sigma x^{2}} d \mu_{\alpha}(\sigma)$.

\section{Diffusion semi-groups}

In this section, we consider a symmetric diffusion semi-group as considered by Stein in [19]:

Definition 3.1. A symmetric diffusion semi-group with infinitesimal generator $L$ is a family of operators $\left(e^{t L}\right)_{t \geq 0}$ such that:

i) $e^{t L}$ is self-adjoint for $t \geq 0$.

ii) $e^{t L}$ is the convolution operator with a probability density function $p_{t}(x)$ $\left(p_{t}(x) \geq 0\right.$ and $\left.\int p_{t}(x) d x=1\right)$.

iii) $e^{t L} e^{s L}=e^{(t+s) L}$ and, for $f \in L^{2}, \lim _{t \rightarrow 0^{+}}\left\|e^{t L} f-f\right\|_{2}=0$.

We then have

iv) $L f=\lim _{t \rightarrow 0} \frac{1}{t}\left(e^{t L} f-f\right)$ on a dense subspace of $L^{2}$.

v) $\partial_{t} e^{t L} f=L\left(e^{t L} f\right)$.

For classical results on such semi-groups, we refer to the survey of Bakry [1]. A crucial result is that, for a convex function $\phi$, we have Jensen's inequality

$$
\phi\left(e^{t L} f\right) \leq e^{t L} \phi(f),
$$

and, by looking at the derivatives of both terms at $t=0$,

$$
\phi^{\prime}(f) L f \leq L(\phi f) .
$$

When $\phi(t)=t^{2}$, we get $2 f L(f) \leq L\left(f^{2}\right)$ : this is the positivity of the square field operator

$$
\Gamma(f, g)=\frac{1}{2}(L(f, g)-f L(g)-g L(f)) .
$$


For $\phi(t)=|t|^{\gamma}$ with $\gamma>1$, we find $\gamma f|f|^{\gamma-2} L(f) \leq L\left(|f|^{\gamma}\right)$. For $\gamma=p / 2$ with $2<p<+\infty$, we multiply the inequality by $|f|^{p / 2}$ and we integrate. We thus get

$$
p \int f|f|^{p-2} L f d x \leq 2 \int|f|^{p / 2} L\left(|f|^{p / 2}\right) d x=-2 \int\left|\sqrt{-L}\left(|f|^{p / 2}\right)\right|^{2} d x .
$$

We are now going to generalize (3.4) by taking into account the sign of $f$ in the RHS of the inequality:

Theorem 3.2. Let $\left(e^{t L}\right)_{t \geq 0}$ be a symmetric diffusion semi-group. Then:

i) For $2 \leq p<+\infty$, we have the inequality

$$
p \int f|f|^{p-2} L(f) d x \leq \int f|f|^{\frac{p}{2}-1} L\left(f|f|^{\frac{p}{2}-1}\right) d x=-\int\left|\sqrt{-L}\left(f|f|^{\frac{p}{2}-1}\right)\right|^{2} d x .
$$

ii) For $1 \leq p \leq 2$, we have the inequality

$$
4 \int f|f|^{\frac{p}{2}-1} L\left(f|f|^{\frac{p}{2}-1}\right) d x=-4 \int\left|\sqrt{-L}\left(f|f|^{\frac{p}{2}-1}\right)\right|^{2} d x \leq p \int f|f|^{p-2} L(f) d x
$$

where, moreover, $p \int f|f|^{p-2} L(f) d x \leq 0$.

Proof. We use the convex function $\phi(t)=|t|$, and we find $\operatorname{sgn}(f) L(f) \leq L(|f|)$, hence $f L(f) \leq|f| L(|f|)$. We decompose $f$ into $f=f^{+}-f^{-}$with $f^{+}=\frac{f+|f|}{2}$, and we get

$$
f^{+} L\left(f^{-}\right)+f^{-} L\left(f^{+}\right) \geq 0
$$

Integrating (3.7) and using the self-adjointness of $L$ gives $\int f^{+} L\left(f^{-}\right) d x \geq 0$. The case of $f^{+}$and $f^{-}$approximating two Dirac masses at separate points gives then that the distribution kernel $K$ of $L$ satisfies $K(x, y) \geq 0$ away from the diagonal $x=y$, and we get finally that

$$
f^{+} L\left(f^{-}\right)=\int_{x \neq y} K(x, y) f^{+}(x) f^{-}(y) d y \geq 0,
$$

and similarly $f^{-} L\left(f^{+}\right) \geq 0$. In particular, we get that, for $1 \leq p<+\infty$, we have

$$
\int\left(f^{+}\right)^{p-1} L\left(f^{-}\right)+\left(f^{-}\right)^{p-1} L\left(f^{+}\right) d x \geq 0 .
$$

On the other hand, we have that $t \mapsto\left\|e^{t L}\right\|_{p}^{p}$ is nonincreasing, so that (by looking at the derivative at $t=0$ ) we have $p \int f|f|^{p-2} L f d x \leq 0$. This inequality together with (3.9) gives

$$
\begin{aligned}
2 \int\left(f^{+}\right)^{p-1} L\left(f^{+}\right)+\left(f^{-}\right)^{p-1} L\left(f^{-}\right) d x & \leq \int f|f|^{p-2} L(f) d x \\
& \leq \int\left(f^{+}\right)^{p-1} L\left(f^{+}\right)+\left(f^{-}\right)^{p-1} L\left(f^{-}\right) d x
\end{aligned}
$$


and, similarly, we have for $g=f|f|^{\frac{p}{2}-1}, g^{+}=\left(f^{+}\right)^{p / 2}$ and $g^{-}=\left(f^{-}\right)^{p / 2}$,

$$
2 \int g^{+} L\left(g^{+}\right)+g^{-} L\left(g^{-}\right) d x \leq \int g L(g) d x \leq \int g^{+} L\left(g^{+}\right)+g^{-} L\left(g^{-}\right) d x \text {. }
$$

When $p \geq 2$, we write $\left\|e^{t L} f^{+}\right\|_{p}^{p} \leq\left\|e^{t L}\left(g^{+}\right)\right\|_{2}^{2}$ and we get (by looking at the derivative at $t=0)$ that $p \int\left(f^{+}\right)^{p-1} L\left(f^{+}\right) d x \leq 2 \int g^{+} L\left(g^{+}\right) d x$; we have the same inequality for $f^{-}$and $g^{-}$. Thus, (3.10) and (3.11) give (3.5).

When $p \leq 2$, we write $\left\|e^{t L} g^{+}\right\|_{2}^{2} \leq\left\|e^{t L}\left(f^{+}\right)\right\|_{p}^{p}$ and get that $2 \int g^{+} L\left(g^{+}\right) d x$ $\leq p \int\left(f^{+}\right)^{p-1} L\left(f^{+}\right) d x$; we have the same inequality for $f^{-}$and $g^{-}$. Thus, (3.10) and (3.11) give (3.6).

\section{A. and D. Córdoba's inequality and Besov norms}

The semi-group $\left(e^{-t \Lambda^{2 \alpha}}\right)_{t>0}$ is a symmetric diffusion semi-group on $\mathbb{R}^{n}$. The positivity of its kernel is a consequence of the positivity of the heat kernel $e^{t \Delta}$ and of the representation formula given by Corollary 2.2. Thus, Córdoba's inequality (1.6) is just a special case of inequality (3.4). In this section, we shall apply Theorem 3.2 (generalization of $(3.4)$ ) to the semi-group $\left(e^{-t \Lambda^{2 \alpha}}\right)_{t \geq 0}$. Our application will be based on the following easy lemma:

Lemma 4.1. Let $0<\gamma \leq 1$. Then for all $a$ and $b$ in $\mathbb{R}$ we have

$$
\left.|a| a\right|^{\gamma-1}-b|b|^{\gamma-1}|\leq 2| a-\left.b\right|^{\gamma}
$$

Proof. This is obvious if $a b<0$ : if $u v<0$ then $\max (|u|,|v|) \leq|u-v| \leq$ $2 \max (|u|,|v|)$. If $a b \geq 0$, we use the fact that $d_{\gamma}(x, y)=|x-y|^{\gamma}$ is a distance on $\mathbb{R}$ and we write $\left|d_{\gamma}(a, 0)-d_{\gamma}(b, 0)\right| \leq d_{\gamma}(a, b) \mid$.

We may now prove the following extension of Córdoba's inequality, using norms in homogeneous Sobolev and Besov spaces:

Theorem 4.2. (A) Let $0<\alpha<1$ and $2 \leq p<+\infty$. Then there is a positive constant $c_{\alpha, p, n}>0$ such that

$$
\begin{aligned}
c_{\alpha, p, n}\|f\|_{\dot{B}_{p}^{2 \alpha / p, p}}^{p} & \leq\left\|f|f|^{\frac{p}{2}-1}\right\|_{\dot{H}^{\alpha}}^{2}=\int\left|\Lambda^{\alpha}\left(f|f|^{\frac{p}{2}-1}\right)\right|^{2} d x \\
& \leq p \int f|f|^{p-2} \Lambda^{2 \alpha}(f) d x .
\end{aligned}
$$

(B) Let $2 \leq p<+\infty$. Then there is a positive constant $c_{p, n}>0$ such that

$$
\begin{aligned}
c_{p, n}\|f\|_{\dot{B}_{p}^{2 \alpha / p, \infty}}^{p} & \leq\left\|f|f|^{\frac{p}{2}-1}\right\|_{\dot{H}^{1}}^{2}=\int\left|\vec{\nabla}\left(f|f|^{\frac{p}{2}-1}\right)\right|^{2} d x \\
& \leq p \int f|f|^{p-2}(-\Delta f) d x .
\end{aligned}
$$


(C) Let $0<\alpha<1$ and $\max (1,2 \alpha)<p<2$. Then there is a positive constant $C_{\alpha, p, n}>0$ such that

$$
\begin{aligned}
0 \leq p \int f|f|^{p-2} \Lambda^{2 \alpha}(f) d x & \leq 4\left\|f|f|^{\frac{p}{2}-1}\right\|_{\dot{H}^{\alpha}}^{2}=4 \int\left|\Lambda^{\alpha}\left(f|f|^{\frac{p}{2}-1}\right)\right|^{2} d x \\
& \leq C_{\alpha, p, n}\|f\|_{\dot{B}_{p}^{2 \alpha / p, p}}^{p} .
\end{aligned}
$$

Proof. First, we will apply Theorem 3.2 to the symmetric diffusion semi-group $\left(e^{-t \Lambda^{2 \alpha}}\right)_{t \geq 0}:$ (3.5) gives the RHS inequalities in (4.2) and (4.3), while (3.6) gives the LHS inequality in (4.4). Thus, the proof of Theorem 4.2 is reduced to a comparison between a Besov norm and a Sobolev norm.

Besov norms may be defined in various (more or less) equivalent ways. We shall use the characterization of Besov spaces through moduli of continuity. For $\beta \in(0,1)$ and $1 \leq p<\infty$, the norms of $\dot{B}_{p}^{\beta, p}$ may be defined as

$$
\left\{\begin{aligned}
\|f\|_{\dot{B}_{p}^{\beta, p}} & =\left(\iint \frac{|f(x)-f(y)|^{p}}{|x-y|^{n+p \beta}} d x d y\right)^{\frac{1}{p}} \\
\text { and } & \\
\|f\|_{\dot{B}_{p}^{\beta, \infty}} & =\sup _{h \in \mathbb{R}^{n}, h \neq 0} \frac{\|f(x)-f(x+h)\|_{p}}{|h|^{\beta}}
\end{aligned}\right.
$$

Moreover, we have $\dot{H}^{\alpha}=\dot{B}_{2}^{\alpha, 2}$. Thus, the Sobolev norm $\|f\|_{\dot{H}^{\alpha}}$ is equivalent, for $\alpha \in(0,1)$, to $\|f\|_{\dot{B}_{2}^{\alpha, 2}}=\sqrt{\iint \frac{|f(x)-f(y)|^{2}}{|x-y|^{n+2 \alpha}} d x d y}$. For $\alpha=1$, the Sobolev norm $\|f\|_{\dot{H}^{1}}$ is equivalent to $\sup _{h \in \mathbb{R}^{n}, h \neq 0} \frac{\|f(x)-f(x+h)\|_{2}}{|h|}$.

To finish the proof, we use Lemma 4.1. For $p \geq 2$, we take $\gamma=2 / p, a=$ $f(x)|f(x)|^{\frac{p}{2}-1}$, and $b=f(y)|f(y)|^{\frac{p}{2}-1}$, and we get

$$
|f(x)-f(y)|^{p} \leq\left. 2^{p}|f(x)| f(x)\right|^{\frac{p}{2}-1}-\left.f(y)|f(y)|^{\frac{p}{2}-1}\right|^{2}
$$

Using (4.6) and (4.5), we then get the LHS inequalities of (4.2) and (4.3).

For $p<2$, we take $\gamma=p / 2, a=f(x)$ and $b=f(y)$, and we get

$$
\left.|f(x)| f(x)\right|^{\frac{p}{2}-1}-\left.f(y)|f(y)|^{\frac{p}{2}-1}\right|^{2} \leq 4|f(x)-f(y)|^{p} .
$$

Using (4.7) and (4.5), for $2 \alpha / p<1$, we then get the RHS inequality of (4.4).

\section{Frequency gaps}

Let $1<p<+\infty$ and let $f \in L^{p}\left(\mathbb{R}^{n}\right)$ be such that the Fourier transform $\hat{f}$ has no low frequency: $\hat{f}(\xi)=0$ for $|\xi| \leq A$. Then it is well known that the norm of $e^{t \Delta} f$ decays exponentially:

$$
\left\|e^{t \Delta} f\right\|_{p} \leq \frac{1}{c_{p}} e^{-c_{p} t A^{2}}\|f\|_{p}
$$

(see for instance Chemin [3]). But (5.1) contains no information for small $t$ 's: if $t \leq A^{-2} \frac{1}{c_{p}} \ln \frac{1}{c_{p}}$ we have $\left\|e^{t \Delta} f\right\|_{p} \leq\|f\|_{p}$ and $1 \leq \frac{1}{c_{p}} e^{-c_{p} t A^{2}}$. 
In this section, we want to prove a more precise estimate:

$$
\left\|e^{t \Delta} f\right\|_{p} \leq e^{-c_{p} t A^{2}}\|f\|_{p} .
$$

We begin with two classical lemmas:

Lemma 5.1. (A) Let $1 \leq p \leq+\infty$ and let $g \in L^{p}\left(\mathbb{R}^{n}\right)$ be such that the Fourier transform $\hat{g}$ has no low frequency: $\hat{g}(\xi)=0$ for $|\xi| \leq A$. Then, for $1 \leq j \leq n$, $\left\|\frac{\partial_{j}}{\Delta} g\right\|_{p} \leq c A^{-1}\|g\|_{p}$.

(B) Let $1 \leq p \leq+\infty$ and let $f \in L^{p}\left(\mathbb{R}^{n}\right)$ be such that the Fourier transform $\hat{f}$ has no low frequency: $\hat{f}(\xi)=0$ for $|\xi| \leq A$. Then $\|f\|_{p} \leq c A^{-1}\|\vec{\nabla} f\|_{p}$.

(C) Let $1 \leq p \leq+\infty$ and let $f \in L^{p}\left(\mathbb{R}^{n}\right)$ be such that the Fourier transform $\hat{f}$ has no low frequency: $\hat{f}(\xi)=0$ for $|\xi| \leq A$. Then there exists $F_{j} \in L^{p}$ such that $f=\sum_{j=1}^{n} \partial_{j} F_{j}$, with $\left\|F_{j}\right\|_{p} \leq c A^{-1}\|f\|_{p}$.

Proof. (A) is obvious: if $\omega \in \mathcal{D}\left(\mathbb{R}^{n}\right)$ is equal to 1 on the ball $B(0,1 / 4)$ and to 0 outside from the ball $B(0,1 / 2)$, then the function $k_{j}$ whose Fourier transform $\hat{k}_{j}$ is equal to $\hat{k}_{j}(\xi)=-\frac{i \xi_{j}}{\|\xi\|^{2}}(1-\omega(\xi))$ satisfies $k_{j} \in L^{1}$. We have $\frac{\partial_{j}}{\Delta} g=A^{n-1} k_{j}(A x) * g$, so that $\left\|\frac{\partial_{j}}{\Delta} g\right\|_{p} \leq A^{-1}\left\|k_{j}\right\|_{1}\|g\|_{p}$.

For $(\mathrm{B})$ and $(\mathrm{C})$, we just write $f=-\sum_{j=1}^{n} \frac{\partial_{j}}{\Delta} \partial_{j} f=-\sum_{j=1}^{n} \partial_{j} \frac{\partial_{j}}{\Delta} f$.

The following lemma can be found in [11]:

Lemma 5.2. Let $1<p<+\infty$ and let $f$ be a $\mathcal{C}^{1}$ function. If $f \in W^{2, p}\left(\mathbb{R}^{n}\right)$, then we have

$$
-\int f|f|^{p-2} \Delta f d x=(p-1) \int_{f(x) \neq 0}|\vec{\nabla} f|^{2}|f|^{p-2} d x
$$

Proof. For $p \geq 2$, this is obvious. $f|f|^{p-2}$ is $\mathcal{C}^{1}$ and $\partial_{j}\left(f|f|^{p-2}\right)=(p-1)|f|^{p-2} \partial_{j} f$. Thus, (5.3) is a direct consequence of integration by parts.

For $1<p<2$, we approximate $f|f|^{p-2}$ by $g_{\epsilon}=f\left|f^{2}+\epsilon^{2}\right|^{\frac{p-2}{2}}$ with $\epsilon>0$. By dominated convergence, we have $-\int f|f|^{p-2} \Delta f d x=\lim _{\epsilon \rightarrow 0} \int g_{\epsilon}(-\Delta f) d x$. We have $\partial_{j}\left(g_{\epsilon}\right)=\partial_{j} f\left|f^{2}+\epsilon^{2}\right|^{\frac{p-2}{2}}\left(1+(p-2) \frac{f^{2}}{f^{2}+\epsilon^{2}}\right)$. We consider $\omega \in \mathcal{D}\left(\mathbb{R}^{n}\right)$ such that $0 \leq \omega \leq 1$ and $\omega=1$ on $B(0,1)$. Then we have

$$
\begin{aligned}
-\int \partial_{j}^{2} f g_{\epsilon} & =\lim _{R \rightarrow+\infty} \int \partial_{j} f\left(\omega(x / R) \partial_{j} g_{\epsilon}+\frac{1}{R} \partial_{j} \omega(x / R) g_{\epsilon}\right) d x \\
& =\int\left|\partial_{j} f\right|^{2}\left|f^{2}+\epsilon^{2}\right|^{\frac{p-2}{2}}\left(1+(p-2) \frac{f^{2}}{f^{2}+\epsilon^{2}}\right) d x
\end{aligned}
$$

since $\left|\int\right| \partial_{j} f \frac{1}{R} \partial_{j} \omega(x / R) g_{\epsilon} d x \mid \leq R^{-1}\left\|\partial_{j} \omega\right\|_{\infty}\|f\|_{W^{2, p}}^{p}$ (and thus goes to 0 as $R$ goes to $+\infty)$, and since $\partial_{j} f \partial_{j} g_{\epsilon} \geq 0$ (note that $\left.p-1 \leq 1+(p-2) \frac{f^{2}}{x^{2}+\epsilon^{2}} \leq 1\right)$, so that we may apply monotone convergence to $\int \partial_{j} f \partial_{j} g_{\epsilon} \omega(x / R) d x$. We may 
restrict the domain of the integral on the RHS of (5.4) to the set of $x$ such that $f(x) \neq 0$, since the set of $x$ such that $f(x)=0$ and $\partial_{j} f(x) \neq 0$ has Lebesgue measure 0 . Thus, we have

$$
-\int f|f|^{p-2} \Delta f d x=\lim _{\epsilon \rightarrow 0^{+}} \int_{f(x) \neq 0}|\vec{\nabla} f|^{2}\left|f^{2}+\epsilon^{2}\right|^{\frac{p-2}{2}}\left(1+(p-2) \frac{f^{2}}{f^{2}+\epsilon^{2}}\right) d x
$$

Moreover $\epsilon \mapsto\left|r^{2}+\epsilon^{2}\right|^{\frac{p-2}{2}}$ is nonincreasing function of $\epsilon \in[0,+\infty)$ and we may apply again monotone convergence to see that

$$
\lim _{\epsilon \rightarrow 0} \int_{f(x) \neq 0}\left|\partial_{j} f\right|^{2}\left|f^{2}+\epsilon^{2}\right|^{\frac{p-2}{2}} d x=\int_{f(x) \neq 0}\left|\partial_{j} f\right|^{2}|f|^{p-2} d x .
$$

The inequality $|\vec{\nabla} f|^{2}\left|f^{2}+\epsilon^{2}\right|^{\frac{p-2}{2}}\left(1+(p-2) \frac{f^{2}}{f^{2}+\epsilon^{2}}\right) \geq(p-1)|\vec{\nabla} f|^{2}\left|f^{2}+\epsilon^{2}\right|^{\frac{p-2}{2}}$, together with (5.5) and (5.6), gives us that the limit in (5.6) is finite. The inequality $|\vec{\nabla} f|^{2}\left|f^{2}+\epsilon^{2}\right|^{\frac{p-2}{2}}\left(1+(p-2) \frac{f^{2}}{f^{2}+\epsilon^{2}}\right) \leq|\vec{\nabla} f|^{2}\left|f^{2}+\epsilon^{2}\right|^{\frac{p-2}{2}}$, together with (5.5), gives us, by dominated convergence, the equality (5.3).

We may now prove our theorem on frequency gaps:

Theorem 5.3. Let $1<p<+\infty$ and let $f \in L^{p}\left(\mathbb{R}^{n}\right)$ be such that the Fourier transform $\hat{f}$ has no low frequency: $\hat{f}(\xi)=0$ for $|\xi| \leq A$. Then:

(A) If $f \in W^{2, p}$, we have the inequality

$$
c_{p}\|f\|_{p}^{p} \leq A^{-2} p \int f|f|^{p-2}(-\Delta f) d x
$$

where the constant $c_{p}>0$ depends only on $n$ and $p$.

(B) We have the inequality, for all $t \geq 0$,

$$
\left\|e^{t \Delta} f\right\|_{p} \leq e^{-c_{p} A^{2} t}\|f\|_{p}
$$

where the constant $c_{p}>0$ depends only on $n$ and $p$.

(C) For $0<\alpha<1$ and $t \geq 0$, we have the inequality

$$
\left\|e^{-t \Lambda^{2 \alpha}} f\right\|_{p} \leq e^{-c_{\alpha, p} A^{2 \alpha} t}\|f\|_{p}
$$

where the constant $c_{\alpha, p}>0$ depends only on $n, \alpha$ and $p$.

Proof. We may assume (by a density argument) that $f$ is smooth. In order to prove (A), we shall consider separately the cases $p \geq 2$ and $p<2$.

Case $p \geq 2$. We use Lemma 5.1 and write $f=\sum_{j=1}^{n} \partial_{j} F_{j}$. Then we have

$$
\|f\|_{p}^{p}=\sum_{j=1}^{n} \int \partial_{j} F_{j} f|f|^{p-2} d x=-(p-1) \sum_{j=1}^{n} \int \partial_{j} f F_{j}|f|^{p-2} d x
$$


and by Cauchy-Schwarz,

$$
\|f\|_{p}^{p} \leq(p-1)\left(\int|\vec{\nabla} f|^{2}|f|^{p-2} d x\right)^{1 / 2}\left(\int \sum_{j}\left|F_{j}\right|^{2}|f|^{p-2} d x\right)^{1 / 2}
$$

We conclude with Lemma $5.1(\mathrm{C})$ and Lemma 5.2.

Case $p<2$. We use Lemma 5.1 (B) and write $\|f\|_{p} \leq c A^{-1}\|\vec{\nabla} f\|_{p}$. Moreover, when computing the integral $\int|\vec{\nabla} f|^{p} d x$, we may restrict the domain of integration to the set of $x$ such that $f(x) \neq 0$. Then we use Hölder's inequality to get

$$
\int|\vec{\nabla} f|^{p} d x \leq\left(\int_{f(x) \neq 0}|\vec{\nabla} f|^{2}|f|^{p-2} d x\right)^{p / 2}\left(\int_{f(x) \neq 0}|f|^{p} d x\right)^{1-p / 2},
$$

and we conclude with Lemma 5.1 (B) and Lemma 5.2.

Thus, (A) is proved. (B) is a direct consequence of (A): the derivative of $H(t)=$ $\left\|e^{t \Delta} f\right\|_{p}^{p}$ is equal to $p \int e^{t \Delta} f\left|e^{t \Delta} f\right|^{p-2} \Delta\left(e^{t \Delta} f\right) d x$, and the the derivative of $K(t)=$ $e^{-c_{p} A^{2} t}\|f\|_{p}^{p}$ is $-c_{p} A^{2} e^{-c_{p} A^{2} t}\|f\|_{p}^{p}$. (A) gives that $H^{\prime}(t) \leq-c_{p} A^{2} H(t)$; thus, we get, for $J(t)=H(t)-K(t), J^{\prime}(t) \leq-c_{p} A^{2} J(t)$ and $J(t) \leq J(0) e^{-c_{p} A^{2} t}=0$. Thus, $H(t) \leq K(t)$ and $(\mathrm{B})$ is proved.

Finally, (C) is a consequence of (B) and of the representation formulae (2.1) and $(2.2)$ :

$$
\begin{aligned}
\left\|e^{-t \Lambda^{2 \alpha}} f\right\|_{p} & \leq \int_{0}^{\infty}\left\|e^{\sigma t^{1 / \alpha} \Delta} f\right\|_{p}^{p} d \mu_{\alpha}(\sigma) \leq \int_{0}^{\infty} e^{-c_{p} A^{2} \sigma t^{1 / \alpha}}\|f\|_{p} d \mu_{\alpha}(\sigma) \\
& =e^{-\left(c_{p} A^{2} t^{1 / \alpha}\right)^{\alpha}}\|f\|_{p}=e^{-c_{p}^{\alpha} A^{2 \alpha} t}\|f\|_{p} .
\end{aligned}
$$

Thus, (C) is proved.

\section{Band limited functions}

In this section, we shall estimate the decay of $\left\|e^{-t \Lambda^{2 \alpha}} f\right\|_{p}$ by below:

Theorem 6.1. Let $1<p<+\infty$ and let $f \in L^{p}\left(\mathbb{R}^{n}\right)$ be such that the Fourier transform $\hat{f}$ has no high frequency: $\hat{f}(\xi)=0$ for $|\xi| \geq A$. Then:

(A) For $0<\alpha \leq 1$, we have the inequality

$$
A^{-2 \alpha} p \int f|f|^{p-2} \Lambda^{2 \alpha} f d x \leq c_{\alpha, p}\|f\|_{p}^{p},
$$

where the constant $c_{\alpha, p}>0$ depends only on $n$ and $p$.

(B) For $0<\alpha<1$ and $t \geq 0$, we have the inequality

$$
\left\|e^{-t \Lambda^{2 \alpha}} f\right\|_{p} \geq e^{-c_{\alpha, p} A^{2 \alpha}}\|f\|_{p},
$$

where the constant $c_{\alpha, p}>0$ depends only on $n, \alpha$ and $p$.

Proof. The case $p \geq 2$ is easy. The Bernstein inequalities give us that $\left\|\Lambda^{2 \alpha}(\theta)\right\|_{p} \leq$ $c A^{2 \alpha}\|\theta\|_{p}$, and thus (6.1) is obvious. 
When $p<2$, we use Theorem 3.2 (equation (3.6)) (or the LHS of Theorem 4.2, equation (4.4), which is valid for $1<p<2$ ), and get that

$$
p \int f|f|^{p-2} \Lambda^{2 \alpha}(f) d x \leq 4\left\|f|f|^{\frac{p}{2}-1}\right\|_{\dot{H}^{\alpha}}^{2} \leq 4\|f\|_{p}^{(1-\alpha) p / 2}\left\|\vec{\nabla}\left(f|f|^{\frac{p}{2}-1}\right)\right\|_{2}^{2 \alpha} .
$$

We approximate $f|f|^{\frac{p-2}{2}}$ by $g_{\epsilon}=f\left|f^{2}+\epsilon^{2}\right|^{\frac{p-2}{4}}$, with $\epsilon>0$. We have $\partial_{j} g_{\epsilon}=$ $\partial_{j} f\left|f^{2}+\epsilon^{2}\right|^{\frac{p-2}{4}}\left(1+\frac{p-2}{2} \frac{f^{2}}{f^{2}+\epsilon^{2}}\right)$. We have that

$$
\begin{aligned}
\left\|\vec{\nabla} g_{\epsilon}\right\|_{2}^{2} & =\int_{f(x) \neq 0}|\vec{\nabla} f|^{2}\left|f^{2}+\epsilon^{2}\right|^{\frac{p-2}{2}}\left(1+\frac{p-2}{2} \frac{f^{2}}{f^{2}+\epsilon^{2}}\right)^{2} d x \\
& \underset{\epsilon>0}{\longrightarrow}(p-1)^{2} \int_{f(x) \neq 0}|\vec{\nabla} f|^{2}|f|^{p-2} d x .
\end{aligned}
$$

We use Lemma 5.2 to get that the limit in (6.4) is finite. Thus, we get that $\vec{\nabla}\left(f|f|^{\frac{p}{2}-1}\right) \in L^{2}$ and that (using Bernstein's inequality)

$$
\left\|\vec{\nabla}\left(f|f|^{\frac{p}{2}-1}\right)\right\|_{2}^{2}=-(p-1) \int f|f|^{p-2} \Delta f d x \leq c A^{2}\|f\|_{p}^{p} .
$$

Thus (A) is proved.

(B) is a direct consequence of $(\mathrm{A})$ : the derivative of $H(t)=\left\|e^{-t \Lambda^{2 \alpha}} f\right\|_{p}^{p}$ is equal to $-p \int e^{-t \Lambda^{2 \alpha}} f\left|e^{-t \Lambda^{2 \alpha}} f\right|^{p-2} \Lambda^{2 \alpha}\left(e^{-t \Lambda^{2 \alpha}} f\right) d x$, and the derivative of $K(t)=$ $e^{-c_{\alpha, p} A^{2} t}\|f\|_{p}^{p}$ is $-c_{\alpha, p} A^{2} e^{-c_{\alpha, p} A^{2} t}\|f\|_{p}^{p}$. (A) gives that $H^{\prime}(t) \geq-c_{\alpha, p} A^{2} H(t)$; thus, we get, for $J(t)=H(t)-K(t)$, the inequalities $J^{\prime}(t) \geq-c_{\alpha, p} A^{2} J(t)$ and $J(t) \geq$ $J(0) e^{-c_{p} A^{2} t}=0$. Thus, $H(t) \geq K(t)$, and $(\mathrm{B})$ is proved.

\section{Danchin's inequality}

In this section, we shall discuss the nonlinear Bernstein inequality given by Danchin in [8] and [9]: for $\theta \in L^{p}\left(\mathbb{R}^{n}\right)$ such that its Fourier transform $\hat{\theta}(\xi)$ is supported in the annulus $1 / 2 \leq|\xi| \leq 2$, we have, for $1<p<\infty$,

$$
A\|\theta\|_{p}^{p} \leq\left\|\vec{\nabla}\left(|\theta|^{p / 2}\right)\right\|_{2}^{2} \leq B\|\theta\|_{p}^{p},
$$

where the constants $A$ and $B$ are positive and depend only on $p$ and on the dimension $n$. Danchin [8] proved it for $p \in 2 \mathbb{N}^{*}$, then Planchon [14] proved it for $p \geq 2$, and finally Danchin gave a proof for $p>1$ in [9]. We shall use our previous results to prove it and generalize it:

Theorem 7.1. Let $1<p<+\infty$. Let $\theta \in L^{p}\left(\mathbb{R}^{n}\right)$ be such that its Fourier transform $\hat{\theta}(\xi)$ is supported in the annulus $1 / 2 \leq|\xi| \leq 2$. Then, for $0<\alpha \leq 1$, we have

$$
A\|\theta\|_{p}^{p} \leq\left\|\Lambda^{\alpha}\left(\theta|\theta|^{p / 2-1}\right)\right\|_{2}^{2} \leq B\|\theta\|_{p}^{p},
$$

where the constants $A$ and $B$ are positive and depend only on $p$, on $\alpha$, and on the dimension $n$. 
Proof. Due to the spectral localization of $\theta$, we have

$$
\|\theta\|_{p} \sim\|\theta\|_{\dot{B}_{p}^{2 \alpha / p, p}} \sim\|\theta\|_{\dot{B}_{p}^{2 \alpha / p, \infty}}
$$

The case $p \geq 2$ is easy: (7.3) and Theorem 4.2 give that $A\|\theta\|_{p}^{p} \leq\left\|\Lambda^{\alpha}\left(\theta|\theta|^{p / 2-1}\right)\right\|_{2}^{2}$. On the other hand, the Bernstein inequalities give us that $\left\|\Lambda^{2 \alpha}(\theta)\right\|_{p} \leq B p^{-1}\|\theta\|_{p}$ so that, using Theorem 4.2 again, we have $\left\|\Lambda^{\alpha}\left(\theta|\theta|^{p / 2-1}\right)\right\|_{2}^{2} \leq p \int \theta|\theta|^{p-2} \Lambda^{2 \alpha}(\theta) d x$ $\leq B\|\theta\|_{p}^{p}$.

When $p \leq 2$, we use Theorem 5.3. We have $\left\|e^{-t \Lambda^{2 \alpha}} f\right\|_{p}^{p} \leq e^{-c_{\alpha, p} t}\|f\|_{p}^{p}$. Looking at the derivatives at $t=0$ (and using Theorem 4.2), we get

$$
c_{\alpha, p}\|f\|_{p}^{p} \leq p \int f|f|^{p-2} \Lambda^{2 \alpha} f d x \leq 4 \int\left|\Lambda^{\alpha}\left(f|f|^{\frac{p}{2}-1}\right)\right|^{2} d x .
$$

On the other hand, (6.3) and (6.5) give us the converse inequality.

Remark. Theorem 7.1 has been proved for $p \geq 2$ by Wu in [21], and Chen, Miao and Zhang in [4].

\section{Lie groups of polynomial growth}

Since our method is mainly based on the use of symmetric diffusion semigroups, our results may be adapted to various settings. In this section, we consider a connected Lie group $G$ and its Lie algebra $\mathcal{G}$, generated from a set of left-invariant vector fields $\left(X_{i}\right)_{1 \leq i \leq N}$ (in the sense of Hörmander: $\mathcal{G}$ is generated by the fields $X_{i}$ and their successive Lie brackets). We consider $d x$ a left-invariant Haar measure on $G$.

We have a Carnot-Carathéodory metric $\rho(x, y)=\left|y^{-1} \cdot x\right|_{G}$ on $G$ associated to the vector fields $X_{i},[7]$. We write $B(x, r)$ for the radius $r>0$ ball centered at $x \in G$, and $V(r)$ for the volume of the ball, $V(r)=\int_{|y|_{G}<r} d y$. The volume obeys to two dimensional orders: for $r<1$, we have $a r^{d} \leq V(r) \leq b r^{d}$ for some local dimension $d>0$ and positive constants $a, b$; for $r \geq 1$, either $V$ has a finite dimensional behaviour, $a r^{D} \leq V(r) \leq b r^{D}$ for some $D>0$ (the dimension at infinity), or $V$ grows exponentially, $e^{a r} \leq V(r) \leq e^{b r}$. In the first case, $G$ is called a group with polynomial growth (versus exponential growth in the second case).

The sublaplacian on $G$ is the operator $\mathcal{J}=-\sum_{i=1}^{N} X_{i}^{2}$. We define the convolution on $G$ by $f * h(x)=\int_{G} f\left(x y^{-1}\right) h(y) d y=\int_{G} f(y) h\left(y^{-1} x\right) d y$. Then $\left(e^{-t \mathcal{J}}\right)_{t \geq 0}$ is a semi-group of positive self-adjoint convolution operators on $G$, so that the theory of symmetric diffusion semigroups can be applied.

We can define Sobolev and Besov spaces on $G$, [20]. When $p=2$, the Besov space $\dot{B}_{2}^{s, 2}$ coincides with the Sobolev space $\dot{H}^{s}=\mathcal{D}\left(\mathcal{J}^{s / 2}\right)$ (normed by $\|f\|_{\dot{H}^{s}}=$ $\left\|\mathcal{J}^{s / 2} f\right\|_{2}$ ). It is easy to check that Saka's characterization of Besov spaces [16] on stratified Lie groups can be extended to the setting of Lie groups with polynomial growth. More precisely, L. Saloff-Coste [17] proved the following result: 
Proposition 8.1. Let $G$ be a connected Lie group with polynomial growth. For $0<s<1$ and $1 \leq p<+\infty$, the norm of the Besov space $\dot{B}_{p}^{s, p}$ is equivalent to

$$
\|f\|_{\dot{B}_{p}^{s, p}}=\left(\iint \frac{|f(x . y)-f(y)|^{p}}{|y|_{G}^{s p} V\left(|y|_{G}\right)} d x d y\right)^{1 / p}
$$

Now, a direct adaptation of Theorem 4.2 gives:

Theorem 8.2. Let $\mathcal{J}$ be the sublaplacian operator on a connected Lie group $G$ with polynomial growth. Let $0<\alpha<1$ and $2 \leq p<+\infty$. Then there is a positive constant $c_{\alpha, p, G}>0$ such that

$$
\begin{aligned}
c_{\alpha, p, G}\|f\|_{\dot{B}_{p}^{2 \alpha / p, p}}^{p} \leq\left\|f|f|^{\frac{p}{2}-1}\right\|_{\dot{H}^{\alpha}}^{2} & =\int\left|\mathcal{J}^{\alpha / 2}\left(f|f|^{\frac{p}{2}-1}\right)\right|^{2} d x \\
& \leq p \int f|f|^{p-2} \mathcal{J}^{\alpha}(f) d x .
\end{aligned}
$$

\section{References}

[1] BAKRy, D.: Functional inequalities for Markov semigroups. In Probability measures on groups: recent directions and trends, 91-147. Tata Inst. Fund. Res., Mumbai, 2006.

[2] Bernstein, S. N.: Sur les fonctions absolument monotones. Acta Math. 52 (1929), no. 1, 1-66.

[3] Chemin, J. Y.: About the Navier-Stokes system. Prépublication du Laboratoire d'Analyse Numérique de Paris 6, 1996.

[4] Chen, Q., Miao, Ch. And Zhang, Z.: A new Bernstein's inequality and the 2D dissipative quasi-geostrophic equation. Commun. Math. Phys. 271 (2007), no. 3, 821-838.

[5] Constantin, P., Majda, A. And Tabak, E.: Formation of strong fronts in the 2-D quasigeostrophic thermal active scalar. Nonlinearity 7 (1994), no. 6, 1495-1533.

[6] Córdoba, A. And Córdoba, D.: A maximum principle applied to quasigeostrophic equations. Commun. Math. Phys. 249 (2004), no. 3, 511-528.

[7] Coulhon, Th., Salof-Coste, L. And Varopoulos, N. Th.: Analysis and geometry on groups. Cambridge Tracts in Mathematics 100, Cambridge University Press, Cambridge, 1992.

[8] Danchin, R.: Poches de tourbillon visqueuses. J. Math. Pures Appl. (9) 76 (1997), no. 7, 609-647.

[9] Danchin, R.: Local theory in critical spaces for compressible viscous and heat-conductive gases. Comm. Partial Differential Equations 26 (2001), no. 7-8, 1183-1233; Erratum: Comm. Partial Differential Equations 27 (2002), no. 11-12, $2531-2532$.

[10] Ju, N.: The maximum principle and the global attractor for the dissipative 2D quasi-geostrophic equations. Commun. Math. Phys. 255 (2005), no. 1, 161-181.

[11] Kato, T.: Liapunov functions and monotonicity in the Navier-Stokes equation. In Functional-analytic methods for partial differential equations (Tokyo, 1989), 53-63. Lectures Notes in Mathematics 1450, Springer, Berlin, 1990. 
[12] Marchand, F.: Existence and regularity of weak solutions to the quasi-geostrophic equations in the spaces $L^{p}$ or $\dot{H}^{-1 / 2}$. Commun. Math. Phys. 277 (2008), no. 1, 45-67.

[13] Pedlosky, J.: Geophysical fluid dynamics. Spinger-Verlag, New York, 1987.

[14] Planchon, F.: Sur un inégalité de type Poincaré. C. R. Acad. Sci. Paris Sér. I Math. 330 (2000), no. 1, 21-23.

[15] Resnick, S.: Dynamical problem in nonlinear advective partial differential equations. Ph.D. Thesis, University of Chicago, 1995.

[16] SakA, K.: Besov spaces and Sobolev spaces on a nilpotent Lie group. Tohoku Math. J. (2) 31 (1979), no. 4, 383-437.

[17] Salof-Coste, L.: Analyse sur les groupes de Lie à croissance polynômiale. Ark. Mat. 28 (1990), no. 2, 315-331.

[18] Samorodnotsky, G. And TaqQu, M. S.: Stable non-Gaussian random processes. Stochastic Modeling. Chapman \& Hall, New York, 1994.

[19] Stein, E. M.: Topics in harmonic analysis related to the Littlewood-Paley theory. Annals of Mathematics Studies 63, Princeton University Press, Princeton, NJ; University of Tokyo Press, Tokyo, 1970.

[20] Triebel, H.: Theory of function spaces II. Monographs in Mathematics 84, Birkhäuser Verlag, Basel, 1992.

[21] Wu, J.: Lower bounds for an integral involving fractional Laplacians and generalized Navier-Stokes equations in Besov spaces. Comm. Math. Phys. 263 (2006), no. 3, 803-831.

[22] Zolotarev, V. M.: One-dimensional stable distributions. Translations of Mathematical Monographs 65, American Mathematical Society, Providence, RI, 1986.

Received December 10, 2010.

Diego Chamorro: Équipe Analyse et Probabilités, EA2172, Université d'Évry Val d'Essonne, 91037 Évry Cedex, France.

E-mail: diego.chamorro@univ-evry.fr

Pierre Gilles Lemarié-Rieusset: Équipe Analyse et Probabilités, EA2172, Université d'Évry Val d'Essonne, 91037 Évry Cedex, France.

E-mail: plemarie@univ-evry.fr 